\title{
Impact of Catechol-O-Methyltransferase on Prefrontal Brain Functioning in Schizophrenia Spectrum Disorders
}

\author{
Ann-Christine Ehlis*,', Andreas Reif', Martin J Herrmann', Klaus-Peter Lesch' and Andreas J Fallgatter' \\ 'Department of Psychiatry and Psychotherapy, University of Wuerzburg, Wuerzburg, Germany
}

\begin{abstract}
The enzyme catechol-O-methyltransferase (COMT) has attracted increasing interest regarding a genetic disposition towards schizophrenias and as a modulator of prefrontal brain function. A common SNP in the COMT gene causes a Val to Met transition at AAI58/AAI08 (Vall58Met), resulting in reduced COMT activity in Met allele carriers. An impact of COMT genotype on cognition has been well established; however, the exact nature of this influence has yet to be elucidated. The aim of this study was to determine whether COMT genotype affects an electrophysiological marker of prefrontal activation and neuropsychological frontal lobe measures in schizophrenia. To this end, 56 acutely psychotic in-patients with schizophrenia spectrum disorders were investigated. Patients with the COMT 1947AA (Met/Met) genotype $(n=13)$ were compared to a carefully matched sample of patients with a GI947A (Val/Met) genotype $(n=15)$; matching criteria included patients' age, handedness, gender distribution, diagnosis, and medication status. A small group of six homozygous Val allele carriers was additionally included to allow an assessment of possible gene-dosage effects. P300 amplitudes and latencies, as well as an electrophysiological marker of prefrontal brain function (NoGo-Anteriorization/NGA) and neuropsychological measures (Stroop Test, Verbal Fluency, Trail Making Test) were regarded. Homozygous Met allele carriers had significantly increased NGA values and fronto-central Nogo amplitudes compared to patients with at least one Val allele. They also tended to perform better in the Stroop task, as compared to the matched group of Val/Met patients. These results indicate that COMT genotype exerts a strong impact on prefrontal functioning and executive control in schizophrenia spectrum disorders.

Neuropsychopharmacology (2007) 32, 162-170. doi:10.1038/s.npp. I30I I5I; published online 28 June 2006
\end{abstract}

Keywords: COMT (catechol-O-methyltransferase); schizophrenia; prefrontal cortex; anterior cingulate cortex (ACC); response control; executive functions

\section{INTRODUCTION}

Schizophrenia is an etiologically heterogeneous group of disorders that involve serious alteration of the patients' cognitive, emotional, and social functioning. One of their cardinal characteristics is a hypofunctionality of the frontal cortex ('hypofrontality concept'; Ingvar and Franzen, 1974), which has been demonstrated in various studies and with different methodological approaches (eg Andreasen et al, 1992; Fallgatter and Mueller, 2001). One of the affected areas is the anterior cingulate cortex (ACC), which has been shown to be deficient at rest (Tamminga et al, 1992) as well as during activation with neuropsychological tasks (Andreasen et al, 1992; Carter et al, 1997). The ACC is critically involved in executive functions such as the monitoring

\footnotetext{
*Correspondence: Dr A-C Ehlis, Laboratory for Psychophysiology and Functional Imaging, Department of Psychiatry and Psychotherapy, University of Wuerzburg, Fuechsleinstrasse 15, Wuerzburg 97080, Germany, Tel: +49 931 201 77410, Fax: +49 931 20I 77550, E-mail: Ehlis_A@klinik.uni-wuerzburg.de

Received 28 November 2005; revised 13 April 2006; accepted 17 May 2006

Online publication: 2 June 2006 at http://www.acnp.org/citations/ Npp060206050698/default.pdf
}

and regulation of ongoing actions, which usually comprise initiation of appropriate and inhibition of inappropriate actions. Tasks that typically involve both these processes are Go-Nogo paradigms that demand the preparation and execution of responses to predefined target stimuli (Go) as well as the inhibition of prepared motor responses (Nogo).

Over the past years, an electrophysiological parameter has been developed and validated that supposedly reflects activation within prefrontal brain areas, particularly involving the ACC (NoGo-anteriorization, NGA; Fallgatter et al, 1997). The NGA quantifies the anteriorization of the positive brain electrical field during the inhibition of prepared (motor) responses that is usually observed during Go-Nogo tasks such as the Continuous Performance Test (CPT). Within the event-related potential (ERP), a marked positive component (P300) can be observed about $300 \mathrm{~ms}$ after presentation of a stimulus, for both Go and Nogo conditions of such a task. However, the topography of the P300 is usually located significantly more anterior during Nogo (response inhibition) as compared to Go (response execution) trials (eg Bokura et al, 2001). This anteriorization of the brain electrical field can now be quantified by the NGA, which represents the geometrical distance (or the arithmetical difference) between the center of gravity 
('centroid') of the Go and Nogo P300 field distribution. In healthy subjects, the NGA was shown to have a very high interindividual stability, excellent short- and long-term test-retest reliability, and it appears to be independent of the subjects' age and gender (reviewed by Fallgatter, 2001). In accordance with the results obtained by brain imaging studies (de Zubicaray et al, 2000; Rubia et al, 2001; Ford et al, 2004; Matthews et al, 2004), electrophysiological source localizations (LORETA procedure; Pascual-Marqui et $a l, 1994)$ have revealed a close relationship between the NGA and a Nogo hyperactivity within prefrontal brain areas, particularly the ACC, in healthy subjects (Strik et al, 1998; Fallgatter et al, 2002). Based on such findings, the NGA has been suggested to be an electrophysiological correlate of cognitive response control and a neurophysiological marker of ACC function, even though other brain regions, particularly within the prefrontal cortex, are likely to be involved as well (eg Ford et al, 2004). In accordance with the hypofrontality concept, schizophrenic patients were found to have a significantly diminished NGA (Fallgatter and Mueller, 2001) and a reduced activation of the ACC during Nogo conditions (Fallgatter et al, 2003), findings that are again largely in line with electrophysiological (Kopp and Rist, 1999; Strandburg et al, 1999; Kiehl et al, 2000; Weisbrod et al, 2000; Mathalon et al, 2002) and neuroimaging data (Volz et al, 1999; Carter et al, 2001; Rubia et al, 2001; Laurens et al, 2003).

The enzyme catechol-O-methyltransferase (COMT) has gathered increasing interest in recent years with respect to the genetic disposition towards schizophrenia and as a modulator of prefrontal function in schizophrenic patients and healthy controls. COMT degrades catecholamines and is thought to compete for dopamine removal from the synaptic cleft with the dopamine transporter. As the latter is abundantly expressed in the striatum, COMT does not seem to play a major role here; however, in the prefrontal cortex, COMT is thought to be crucially involved in dopamine metabolism (Weinberger et al, 2001).

Schizophrenia has a substantial heritability of $>80 \%$, as estimated in a recent meta-analysis (Sullivan et al, 2003). Thus, huge efforts have been made to identify disease genes. Chromosome 22q has been described as one of the confirmed regions for susceptibility loci (Badner and Gershon, 2002). Interestingly, the gene encoding COMT resides at 22q11, which makes it an attractive candidate gene. A common SNP was found in the COMT gene causing a Val to Met transition at amino-acid position 158 (or 108, respectively), which is commonly designated as Val158Met. The Met allele codes for a 'thermo-labile' enzyme displaying lower enzymatic activity, resulting in increased synaptic dopamine and strengthened (prefrontal) dopaminergic tone. Met/Met homozygotes display approximately $25 \%$ COMT activity compared to Val/Val homozygotes, with heterozygotes in-between. COMT was therefore one of the prime candidate genes to identify disease genes for psychosis, and Val158Met is currently one of the most frequently studied polymorphisms in schizophrenia. The results of association studies, however, are somewhat ambiguous with both positive (eg Wonodi et al, 2003) and negative (Inada et al, 2003) findings. A recent haplotype analysis (Sanders et al, 2005) added further evidence for COMT making a contribution to the genetic risk of schizophrenia, as did a study utilizing a large number of Irish schizophrenia high-density families (Chen et al, 2004). Importantly, in both cases, the over-transmitted haplotype included the Val allele.

At a functional level, the impact of COMT genotype on cognition has gathered increasing interest in recent years. Evidence for the influence of COMT on cognitive abilities came from COMT knockout mice that were found to display improved performance in a memory task (Kneavel et al, 2000). Val158Met thereafter has been repeatedly examined regarding its association with cognitive functioning, and in a seminal study Weinberger and associates showed that the Val allele causes reduced performance in the Wisconsin Card Sorting Test (thought to mirror frontal executive functioning) in schizophrenic patients as well as healthy controls (Egan et al, 2001), a finding that could be replicated in numerous subsequent studies. Regarding other neuropsychological functions, Val158Met was also found to influence processing speed and attention in chronic schizophrenic patients (Bilder et al, 2002) and working memory in schizophrenics, their siblings, and controls (Goldberg et al, 2003). In a recent study (Nolan et al, 2004), schizophrenic Met allele carriers displayed better cognitive stability, whereas subjects with the Val allele showed better cognitive flexibility.

Thus, COMT apparently plays a role in some cognitive domains, although its exact contribution to functional skills has yet to be elucidated. One approach to further clarify the meaning of COMT for cognitive functioning and underlying cerebral mechanisms is the examination of neurophysiological parameters that reflect basic mechanisms of brain activation during the performance of cognitive operations and that are likely to be more directly linked to underlying genomic variation than a highly variable behavioral phenotype. Compared to association studies with genetically complex behavioral traits, which frequently comprise several hundreds of subjects, robust gene-brain activity correlations allow the investigation of substantially smaller sample sizes (Egan et al, 2001; Fallgatter et al, 2004). As the NGA has been suggested to be an electrophysiological correlate of prefrontal functioning and is closely related to fundamental cognitive processes of executive control, it might be a promising research parameter in attempts to further examine the impact of COMT genotype on such processes. Considering the above-mentioned findings, we reasoned that COMT genotype might influence this electrophysiological marker in schizophrenia spectrum disorders. Therefore, the NGA was recorded in 34 carefully selected psychotic patients and correlated to Val158Met.

\section{PATIENTS AND METHODS}

\section{Patients}

A total of 56 acutely psychotic psychiatric in-patients who were suffering from schizophrenia spectrum disorders were investigated. Exclusion criteria were age below 18 and above 60 years, comorbidity with other currently present axis-I disorders, a history of or an actually manifest disease of the CNS, or other severe somatic diseases. Thirteen patients with a COMT 1947AA genotype (homozygous Met allele carriers) had a sufficient number of at least 20 artifact-free 
ERP epochs, and could therefore be included in the present analysis (eight male, 10 right-handed, mean age $39.8 \pm 7.0$ years). To ensure proper matching, 15 out of 22 patients with a G1947A genotype (Val/Met allele carriers) were selected to closely resemble the AA sample in age, handedness, and gender distribution (seven male, 13 right-handed, 38.6 \pm 11.4 years), as well as diagnoses and medication status (antipsychotics and co-medication, see below). The matching procedure was performed meticulously and before all data analyses. As a result, the selected group of $\mathrm{Val} / \mathrm{Met}$ carriers did not differ significantly from the Met/Met group in any of the above-mentioned parameters $\left(t_{\mathrm{age}}=0.34, p=0.74 ; t_{\mathrm{cpz}}\right.$ (daily chlorpromazine equivalents in $\mathrm{mg}$ (see below) $)=1.05, p=0.30$; all $\chi^{2}<0.8$, $p>0.4)$. A small group of six homozygous Val allele carriers (three male, six right-handed, mean age $30.7 \pm 9.3$ years) was additionally included to allow an assessment of genedosage effects, at least on a descriptive level. As this last group was very small and thus could not be strictly matched to resemble the other two genotype groups, it was only included in additional non-parametric statistical analyses (see below), which should be regarded as exploratory.

According to the SKID-I-Interview, patients were diagnosed as disorganized $(295.10 ; n=6)$, catatonic (295.20; $n=4)$, paranoid $(295.30 ; n=11)$, and undifferentiated $(295.90 ; n=3)$ types of schizophrenia, schizophreniform $(295.40 ; n=8)$, or schizoaffective disorders $(295.70 ; n=2)$. The mean duration of the disease was $172 \pm 104$ months (mean $\pm S D$ ), with an average of $6.9 \pm 7.5$ admissions to psychiatric hospitals. Five patients had a positive family history of schizophrenia; another 10 patients had firstdegree relatives with non-psychotic or unknown psychiatric conditions. No significant current comorbidities were found in either patient sample except for one patient with neuroleptic-induced adiposity and one with hypertonia; in the Val/Met group, two patients had life-time comorbidities (Bulimia nervosa; anxiety disorder), in the Met/Met group one patient had a previous anorexia nervosa.

Neuroleptic treatment consisted of $529 \pm 400 \mathrm{mg}$ chlorpromazine equivalents per day; 13 patients were treated with typical antipsychotics, 15 patients received atypical antipsychotics, and six patients no neuroleptic medication. As already mentioned above, Val/Met and Met/Met patients did not differ significantly regarding the diagnoses, nor the amount or type of antipsychotic medication. Comedications were regarded in the matching procedure as well, so that the mean daily doses did not differ significantly between the two genotypes $(t<1.2, p>0.25)$ for any of the potentially relevant substance groups (carbamazepin, biperiden, lithium, lorazepam, valproic acid, SSRI).

Written informed consent was obtained from all patients after the procedures had been fully explained. The study was approved by the Ethics Committee of the University of Wuerzburg, and the procedures involved were in accordance with the Declaration of Helsinki.

\section{Psychopathological and Neuropsychological Assessment}

Each patient underwent an extensive psychometric examination, consisting of the SKID-I-Interview, the Brief Psychiatric Rating Scale (BPRS; Overall and Gorham, 1962), the Positive and Negative Symptoms Scale (PANSS;
Kay, 1991), and the Hamilton Depression Rating Scale (HDRS; Hamilton, 1960).

The neuropsychological assessment consisted of the Verbal Fluency Test (VFT), a Stroop Color Word Task, and the Trail Making Test (TMT). For the VFT, patients were instructed to name as many nouns as possible beginning with a certain letter ('letters version') or belonging to a certain category of words ('categories version'). The Stroop task consisted of three parts, with two control conditions ('word reading', 'color naming') and one interference condition (color words were presented in a color that did not correspond to the word meaning of the color word, and patients were instructed to name the ink color of the words). For the TMT, patients had to connect the numbers 1-15, randomly distributed on a sheet of paper, in the correct order (Part A), or connect the numbers $1-8$ and the letters $A-G$ in the correct order while consecutively alternating between numbers and letter (Part B).

\section{Electrophysiological Investigation}

The electrophysiological investigation took place in an electrically shielded, dimly lit room where the participants performed a CPT. Letters were presented sequentially in a pseudo-randomized order and the patients had to press a response button whenever the letter ' $O$ ' (Primer) was directly followed by an ' $\mathrm{X}$ ' (Go condition). The whole stimulus set consisted of 400 letters, with 114 primer stimuli, 57 Go and Nogo conditions ( $\mathrm{O}$ followed by any other letter), and 172 distractors. Each letter was presented for $200 \mathrm{~ms}$ with an interstimulus interval of $1650 \mathrm{~ms}$. The recording sessions took place between 0800 and 1200 .

The EEG was recorded from 21 scalp electrodes placed according to the International 10-20 system (Jasper, 1958) with three additional electrodes to monitor eye movements. Linked mastoids were used as the recording reference; electrode impedances were below $5 \mathrm{k} \Omega$. The recording system involved a 32-channel DC amplifier (Brain Star System) and the Neuroscan data acquisition software calibrated with an external $100 \mu \mathrm{V} / 10 \mathrm{~Hz}$ signal. With an A/D rate of $256 \mathrm{~Hz}$, the hardware filter was set to a bandpass of $0.1-70 \mathrm{~Hz}$.

\section{Data Analysis}

Data were analyzed offline with the program 'Vision Analyzer' (Brain Products, Munich, Germany). After re-referencing the data to an average reference, they were segmented according to the conditions of the CPT (segments from -100 to $700 \mathrm{~ms}$ after stimulus presentation), and the Go and Nogo epochs were further analyzed. A computerized artifact rejection excluded all segments with amplitudes exceeding $\pm 50 \mu \mathrm{V}$; if at least 20 artifact-free EEG epochs were available for the Go and the Nogo condition, the remaining segments were averaged to one Go and one Nogo ERP per patient.

In the individual ERPs, the global field power (GFP; Lehmann and Skrandies, 1980) peaks were determined within a P300 time frame $(275-530 \mathrm{~ms}$; based on a visual inspection of the grand average curves). The GFP represents the mean of all possible potential differences in a given 
scalp potential field and is used as a measure of the amount of activity in this field. At the individual GFP peaks, the amplitude, latency, and anterior-posterior location of the positive centroid (the amplitude-weighted center of gravity of the positive brain electrical field; Lehmann, 1987) were calculated. The centroid locations were quantified by a coordinate system defined by a two-dimensional delineation of the electrode array (cf Figure 1). For the purpose of the present study, only the centroids in the anterior-posterior direction were of interest, the more anterior locations of the centroids being represented by smaller numbers on this axis (eg ' 1 ' represents electrode position Fpz, '5' represents Oz). Finally, the NGA, defined as the distance between the individual Go and Nogo centroid within the coordinate system (unit of the NGA = 'electrode positions'), was calculated.

In addition to this topographical analysis, the P300 peaks were determined for the three midline electrode positions $(\mathrm{Fz}, \mathrm{Cz}, \mathrm{Pz})$ employing a semi-automatic peak picking procedure and using the same time frame mentioned above (275-530 ms post-stimulus); amplitudes and latencies of these peaks were analyzed.

\section{Genotyping}

DNA was extracted from whole blood. Genotyping for COMT G1947A SNP was accomplished using standard PCR procedures modified from a previously published protocol (Egan et al, 2001); primers were $5^{\prime}$-GGG GCC TAC TGT GGC TAC TC- $3^{\prime}$ (forward) and $5^{\prime}$-TTT TTC CAG GTC TGA CAA CG-3' (reverse). Briefly, PCR reactions were performed in a reaction volume of $25 \mu$, including approximately $50 \mathrm{ng}$ of template genomic DNA, $10 \mathrm{pmol}$ of each primer, $2.5 \mathrm{mM}$ of each dNTP, $0.75 \mathrm{mM} \mathrm{MgCl}$, and $1 \mathrm{U}$ of Taq DNA polymerase. Annealing temperature was $58^{\circ} \mathrm{C}$ ( 35 cycles). PCR products were digested with NlaIII $\left(3 \mathrm{~h}\right.$ at $37^{\circ} \mathrm{C}$; fragment sizes: wild-type G1947, $114 \mathrm{bp}$; 1947A variant, 96 and $13 \mathrm{bp}$ ) and subsequently visualized on a $4 \%$ agarose gel. G1947 corresponds to the high-activity Val158 allele; 1947A codes for the low-activity Met variant.

\section{Statistical Analysis}

Only the data of Met/Met and Val/Met carriers were subjected to parametric statistical analyses, as only these two groups were properly matched and included a sufficient number of patients per group. For the P300 centroids of these two groups, a $2 \times 2$ analysis of variance (ANOVA) for repeated measurements was conducted, with the within-subject factor 'condition' (Go, Nogo) and the between-subject factor 'group' (Met/Met vs Val/Met). Post hoc analyses were conducted by means of two-tailed $t$-tests for matched or independent samples. $T$-tests were also used to compare the single electrode (peak) ERP data, the NGA, and the behavioral performance between the two groups. Equality of variances was tested by means of Levene's test, and corrections for inequality were performed whenever necessary. As the number of commission errors was not normally distributed (Kolmogorov-Smirnov $Z=1.54, p<0.05$ ), between-group comparisons for this parameter were conducted by means of Mann-Whitney $U$-tests. All the other electrophysiological and behavioral parameters were normally distributed (Kolmogorov-Smirnov $Z<1.2, p>0.2$ ) and were therefore subjected to parametric testing procedures.
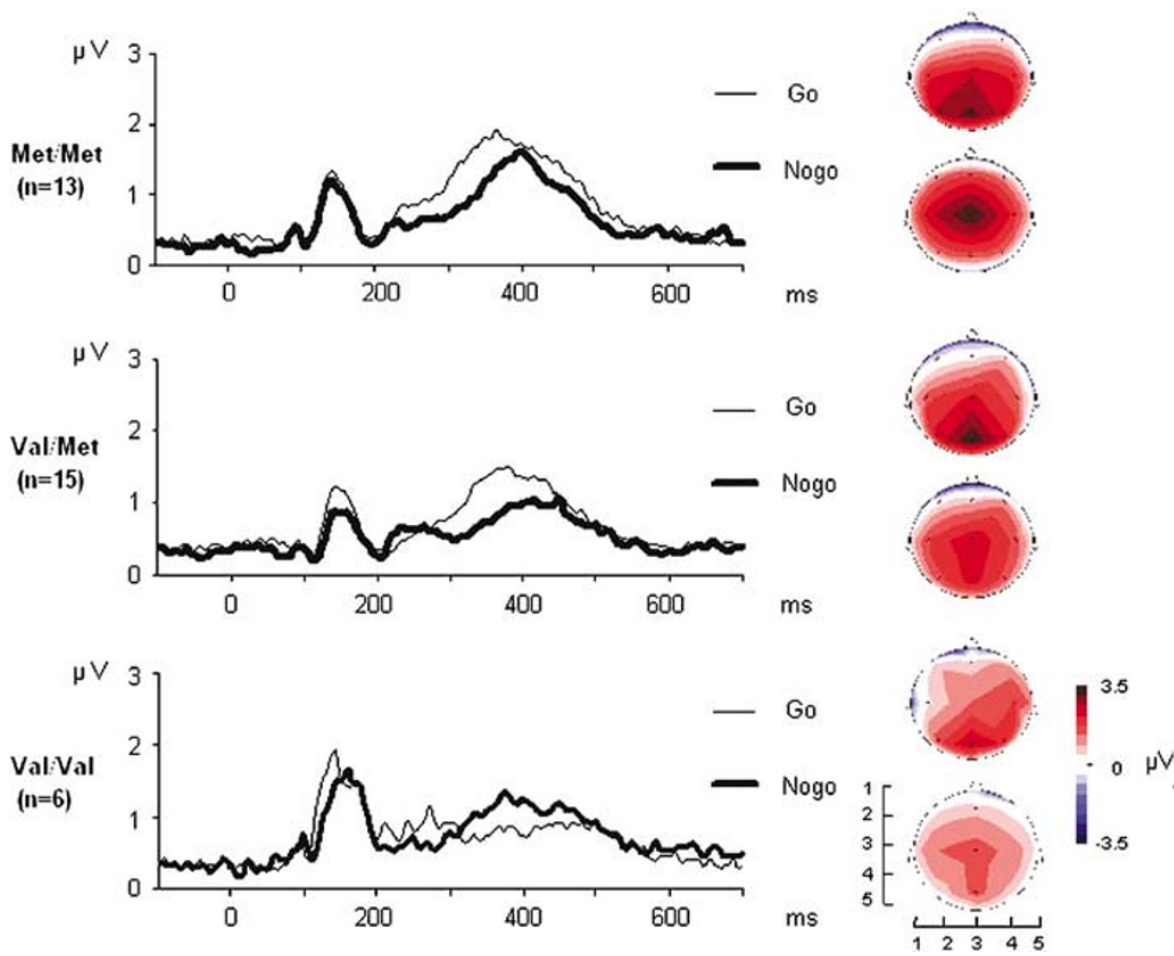

Figure I GFP grand average curves. GFP curves for the CPT Go (thin) and Nogo condition (bold line) in patients with Met/Met ( $n=13$ ), Val/Met $(n=15)$, and $\mathrm{Val} / \mathrm{Val}(n=6)$. The maps display the brain electrical field at the time point of the GFP peak for the Go (the upper one of each pair of maps) and Nogo (lower maps) condition. 
Regarding the third genotype group (Val/Val; $n=6$ ), supplementary non-parametric test procedures were additionally conducted to investigate an effect of the factor 'genotype' on neurophysiological and behavioral parameters across the three COMT polymorphism groups (Kruskal-Wallis tests with Mann-Whitney $U$-tests for post hoc comparisons). Because of the small number of patients included in the third genotype group, however, this additional statistical analysis should be regarded as preliminary and the results should be interpreted carefully.

\section{RESULTS}

\section{Performance Measures, Psychometry, Neuropsychology}

Reaction times, commission errors (button-press after non-target stimulus), and omission errors (no response to Go stimulus) were used as performance measures for the CPT. Statistical testing revealed no significant differences between the two matched groups (Met/Met and Val/Met) for any of the behavioral measures (reaction times: $633 \pm 200 \mathrm{vs}$ $606 \pm 126 \mathrm{~ms}, t_{26}=0.44$, NS; omission errors: $8.6 \pm 9.5 \mathrm{vs}$ $8.5 \pm 9.4$ errors, $t_{26}=0.02$, NS; commission errors: $2.3 \pm 3.3$ vs $1.1 \pm 1.4$ errors, $U=84.5$, NS) (Val/Val: $\mathrm{RT}=580 \pm 86 \mathrm{~ms}$; $3.33 \pm 2.50$ omission errors; $1.83 \pm 2.40$ commission errors; Kruskal-Wallis $\chi^{2}=0.73,1.74$, and 0.44 , respectively; $p>0.4)$. Regarding the different psychometric scales, the two groups did not differ significantly either, even though patients with a Met/Met genotype exhibited slightly higher scores on most of them (Table 1).

Regarding the neuropsychological tests, Met/Met and $\mathrm{Val} /$ Met patients did not differ significantly in their VFT (letters: $21.7 \pm 9.8 v s$ s $24.2 \pm 12.1$ words, $t_{26}=0.60$, NS; categories: $29.1 \pm 10.4$ vs $32.9 \pm 10.5$ words, $t_{25}=0.97$, NS) (Val/Val: $18.5 \pm 7.1$ and $29.0 \pm 10.6$ words, respectively; Kruskal-Wallis $\left.\chi^{2}<3.8, p>0.15\right)$ or TMT performance (A: $28.2 \pm 19.4$ vs $30.7 \pm 12.1 \mathrm{~s}, t_{26}=0.42$, NS; B: $54.9 \pm 34.8$ vs $70.2 \pm 46.0 \mathrm{~s}, t_{26}=0.98$, NS) (Val/Val: $21.0 \pm 7.6$ and $58.0 \pm 31.7 \mathrm{~s}$ in TMT A and B; Kruskal-Wallis $\chi^{2}<3.8$, $p>0.15$ ); however, for the interference condition of the Stroop Test, the group of patients with a heterozygous genotype exhibited a statistical trend for prolonged times compared to the Met/Met group $(116.8 \pm 35.0 \mathrm{vs}$ $\left.146.4 \pm 54.7 \mathrm{~s}, t_{24}=1.73, p<0.1\right)(\mathrm{Val} / \mathrm{Val}$ : Stroop interfer- ence: $138.7 \pm 66.7 \mathrm{~s}$; Kruskal-Wallis $\chi^{2}<3.8, p>0.15$ ). For the two control conditions of the Stroop task, on the other hand, a similar trend could not be detected, the time needed to accomplish the tasks being very similar for all groups (data not shown).

\section{NGA and ERP Data}

Regarding the topographical ERP analysis, the ANOVA for the positive centroids revealed a significant main effect of the factor 'condition' $\left(F_{1,26}=9.18, p<0.01\right)$ and a significant interaction 'condition $\times$ group' $\quad\left(F_{1,26}=5.99\right.$, $p<0.05)$.

Regarding the main effect, the centroid was located more anteriorly in CPT Nogo trials $(3.2 \pm 0.1)$ as compared to Go conditions ( $3.5 \pm 0.1)$, which is a common finding ('NGA') even more markedly visible in healthy subjects. Regarding the interaction, the Nogo-related anteriorization of the brain electrical field was much more pronounced in patients carrying Met/Met (Go vs Nogo centroid: $3.54 \pm 0.66$ vs $\left.3.08 \pm 0.43, t_{12}=3.75, p<0.01\right)$ than in the $\mathrm{Val} / \mathrm{Met}$ group $\left(3.41 \pm 0.62\right.$ vs $3.36 \pm 0.69, t_{14}=0.43$, NS; Figure 1$)$.

This finding is confirmed by a significantly reduced NGA in the group of Val/Met patients compared to the Met/Met group (Table 2). Homozygous Val allele carriers $(n=6)$ showed an even further reduced mean NGA $(-0.37 \pm 1.00)$, a Kruskal-Wallis test confirming an effect of the factor 'genotype' on the NGA across the three groups $\left(\chi^{2}=7.25\right.$, $p<0.05)$ with a significantly larger NGA in Met/Met patients as compared to Val allele carriers (Val/Met: $U=48.0, p<0.05$; Val/Val: $U=15.0, p<0.05)$. Thus, the Val allele caused a reduced anteriorization of the brain electrical field during Nogo conditions in a linear way (gene-dose effect).

These topographical findings were largely reflected by the single electrode ERP data (Table 2). Regarding the two matched groups of patients, Val/Met and Met/Met subjects did not differ significantly regarding any of the ERP measures elicited by Go trials. For the Nogo condition, however, Val/Met patients exhibited significantly decreased P300 amplitudes at fronto-central electrode sites, and it is this failure to generate a robust fronto-central P300 during the CPT Nogo condition that underlies the reduced NGA we observed in this group of patients. When taking into

Table I Psychometric Data

\begin{tabular}{|c|c|c|c|c|c|}
\hline & Val/Val $(n=6)$ & Val/Met $(n=15)$ & Met/Met $(n=13)$ & t (Val/Met vs Met/Met) & Kruskal-Wallis $\left(\chi^{2}\right)$ \\
\hline HDRS & $9.50 \pm 3.99$ & $8.00 \pm 4.83$ & $11.77 \pm 6.85$ & $1.70, N^{\mathrm{a}}$ & $2.21, \mathrm{NS}$ \\
\hline BPRS & $37.00 \pm 6.51$ & $36.80 \pm 8.00$ & $41.54 \pm 13.37$ & $1.12, \mathrm{NS}$ & $0.53, \mathrm{NS}$ \\
\hline PANSS & $56.00 \pm 7.18$ & $57.33 \pm 10.53$ & $67.85 \pm 21.38$ & $1.61, \mathrm{NS}^{\mathrm{a}}$ & I.74, NS \\
\hline Pos & $15.50 \pm 5.17$ & $|2.87 \pm 4.4|$ & $14.92 \pm 4.89$ & I.I7, NS & $2.48, \mathrm{NS}$ \\
\hline GAF & $41.50 \pm 9.05$ & $39.93 \pm 6.86$ & $38.15 \pm 7.16$ & $0.66, \mathrm{NS}$ & 0.48, NS \\
\hline
\end{tabular}

${ }^{\mathrm{a}} 0.1<p<0.2$. 
Table 2 ERP Data

\begin{tabular}{|c|c|c|c|c|c|}
\hline & $\mathrm{Val} / \mathrm{Val}(n=6)$ & Val/Met $(n=15)$ & Met/Met $(n=13)$ & t (Val/Met vs Met/Met) & Kruskal-Wallis $\left(\chi^{2}\right)$ \\
\hline \multicolumn{6}{|l|}{ Go } \\
\hline $\mathrm{Fz}$ & $1.20 \pm 0.75$ & $1.05 \pm 0.84$ & $1.18 \pm 1.28$ & $0.30, \mathrm{NS}$ & $0.13, \mathrm{NS}$ \\
\hline $\mathrm{Cz}$ & $1.73 \pm 1.11$ & $2.68 \pm 1.20$ & $3.22 \pm 1.53$ & I.05, NS & $3.96, \mathrm{NS}$ \\
\hline $\mathrm{Pz}$ & $2.80 \pm 1.12$ & $3.73 \pm 1.20$ & $3.67 \pm 1.85$ & $0.11, N S$ & 2.37, NS \\
\hline GFP & $2.15 \pm 0.79$ & $2.29 \pm 0.74$ & $2.58 \pm 0.91$ & $0.91, \mathrm{NS}$ & I.4I, NS \\
\hline \multicolumn{6}{|l|}{ Nogo } \\
\hline $\mathrm{Fz}$ & $1.49 \pm 0.95$ & $1.28 \pm 0.72$ & $1.96 \pm 0.80$ & 2.37, $p<0.05$ & $3.95, \mathrm{NS}$ \\
\hline $\mathrm{Cz}$ & $2.64 \pm 0.91$ & $2.54 \pm 1.06$ & $3.81 \pm 1.45$ & $2.66, p<0.05$ & $5.01, p<0.1$ \\
\hline $\mathrm{Pz}$ & $2.22 \pm 0.88$ & $2.23 \pm 0.91$ & $2.26 \pm 1.25$ & $0.06, \mathrm{NS}$ & $0.09, \mathrm{NS}$ \\
\hline
\end{tabular}

account the group of Val/Val patients as well, a KruskalWallis test confirmed a statistical trend for a genotype effect on the $\mathrm{Cz}$ amplitude only $\left(\chi^{2}=5.01, p<0.1\right)$, Met/Met patients showing increased amplitudes as compared to Val allele carriers (Val/Met: $U=54.5, p<0.05$; $\mathrm{Val} / \mathrm{Val}$ : $U=19.0, p<0.1)$ All three groups exhibited very similar P300 latencies, with no significant differences between the different genotypes (data not shown).

To ensure that between-group differences were not caused by differences in the number of ERP epochs included in the analyses, the number of artifact-free ERP epochs was determined for each group. With a mean of $43.2 \pm 11.2,43.2 \pm 9.2$, and $49.0 \pm 5.7$ Go epochs, as well as $48.6 \pm 8.1,47.6 \pm 7.6$, and $51.0 \pm 3.0$ Nogo segments included in the analysis of patients with a Met/Met, Val/Met, and Val/Val genotype, respectively, the three groups did not differ significantly in either condition (Go: $F_{2,31}=0.91$; $p=0.41 ;$ Nogo: $F_{2,31}=0.47 ; p=0.63$ ).

\section{DISCUSSION}

We conducted an electrophysiological and neuropsychological assessment of patients with schizophrenia spectrum disorders stratified for COMT Val158Met polymorphism. In contrast to rather small effects on neuropsychological measures, the Val allele impacted heavily on the NGA, an electrophysiological marker of prefrontal brain function. Val allele carriers had a virtually absent anteriorization of the brain electrical field during Nogo trials, indicating reduced activation within prefrontal brain regions during conditions that impose increased demands on cognitive response control. This effect occurred in a genedosage-dependent manner, with Met/Met patients showing the largest, Val/Met patients an intermediate, and Val/Val patients a very small, even negative mean NGA. This indicates that patients with a particularly strong dopaminergic tone showed most consistently the expected functional activation pattern in a task involving prefrontal engagement. This is in accordance with previous studies pointing towards a role of COMT genotype in cognition, particularly with respect to prefrontal brain functions (see Introduction). This topographical effect was also partially reflected in the single electrode ERP data, which showed a modulation by COMT genotype in a very similar way, albeit not as strongly and consistently. In line with the present findings, we showed in a preliminary report that two subjects suffering from 22q11 deletion syndrome and thus hemizygous for COMT featured an absent NGA as well (Reif et al, 2004), further underscoring the results of the present study.

In the present study, only patients with schizophrenia spectrum disorders were investigated. Thus, no statement on the electrophysiological impact of the COMT Val158Met polymorphism in healthy control subjects as compared to schizophrenic patients can be made on the basis of the present data. There are, however, numerous studies reporting a detrimental effect of the Val allele on prefrontal functioning (neuropsychological and neuroimaging data) in both schizophrenic patients and healthy controls (for review see Tunbridge et al, 2006; Craddock et al, 2006). As an underlying explanatory model, Weinberger and colleagues suggested an inverted U-shaped relation between cortical dopamine and prefrontal cortex function, the precise effect of COMT activity depending on the basic dopaminergic tone of a given individual on this $\mathrm{U}$-shaped curve. They furthermore suggest that in healthy controls without pharmacological intervention, individuals with a Met/Met phenotype are located around the peak of the inverted U-shaped curve, with $\mathrm{Val} / \mathrm{Met}$ and $\mathrm{Val} / \mathrm{Val}$ carriers located slightly further down along the curve's rising 'left' arm (Tunbridge et al, 2006). This would account for the replicated finding of a dose-dependent positive influence of the Met allele on prefrontal (cognitive) functioning. Assuming that schizophrenic patients show hyperactive mesolimbic dopamine projections, but hypoactive mesocortical dopamine projections to the prefrontal cortex (eg Abi-Dargham and Moore, 2003), they should generally be 
located further down towards the beginning of the rising part of the inverted U-shaped curve. The principal effect of COMT genotype should therefore be similar in healthy controls as compared to the group of patients investigated here, although it is probably enhanced in schizophrenic patients because of their abnormal dopaminergic state (steeper gradient of the curve towards its beginning). In summary, based on these considerations, healthy controls should be 'superior' to schizophrenic patients regarding their prefrontal functioning irrespective of COMT genotype; but within the group of healthy controls, COMT should exert a similar-albeit weaker-effect as compared to patients with schizophrenia spectrum disorders.

Two previous reports focused on electrophysiological measures as a function of COMT genotype. Tsai et al (2003) examined healthy female subjects and found that Met allele carriers had significantly reduced P300 latencies in a genedose-dependent manner. Gallinat et al (2003), on the other hand, investigated schizophrenic patients and healthy controls by means of an auditory oddball paradigm without finding an effect of COMT genotype on P300 latencies. However, fronto-central P300 amplitudes were lower in 158 Met homozygous subjects, particularly in schizophrenia. This led the authors to the conclusion that in schizophrenics homozygous for the Met allele, less prefrontal cortical 'noise' occurred, which might be involved in the superior performance of Met allele carriers with regard to working memory and information processing. As the authors employed a paradigm in which the frontal component of the P300 can be considered as a correlate of cortical noise, whereas other tasks (such as the CPT) involve frontal P300 components with strong 'signal' properties, the results reported by Gallinat et al do not contradict the present data. In fact, both their results and our own findings indicate better prefrontal functioning in Met allele carriers, whereat two different correlates of prefrontal brain function were used (electrophysiologically assessed prefrontal noise vs ERP components related to prefrontal inhibitory control).

Regarding the psychopathological data, patients homozygous for the Met allele tended to be more severely ill, which is in accordance with previous studies (Bilder $e t$ al, 2002). For two of the three neuropsychological tests of frontal lobe function, no influence of COMT genotype was found, whereas for the Stroop Test, a statistical trend indicated prolonged interference times in patients carrying $\mathrm{Val} / \mathrm{Met}$ as compared to the matched group of Met/Met patients. As both the Stroop interference condition (Gruber et al, 2002) and the NGA (Fallgatter et al, 2002) have been associated with ACC functioning and our two groups of patients differed regarding their mean NGA, an accompanying difference in Stroop interference performance is highly plausible. As the Stroop task was the only task we used that is thought to specifically involve the ACC, a missing effect in the other two tests - which have been shown to preferentially activate other frontal areas such as the dorsolateral prefrontal cortex or Broca's area (Gaillard et al, 2000; Moll et al, 2002) - appears to be plausible as well.

Based on the electrophysiological findings, one might expect Val allele carriers to display an increased CPT error rate, particularly during Nogo trials (ie commission errors).
The reason for the absence of such a finding might well be the paradigm used: The version of the CPT employed in the present study was adapted for application in psychiatric samples, that is, with low difficulty to avoid high error rates, making it less sensitive for respective performance differences.

When interpreting the present findings, it has to be considered that the patient sample was diagnostically heterogeneous, as not only patients with narrow-definition schizophrenia but also some patients with schizophreniform or schizoaffective disorders were included. Although these diagnoses were equally distributed across the different genotype groups (see Patients and methods) so that the observed group differences cannot be attributed to diagnostic between-group differences, it cannot be ruled out that the impact of COMT genotype on prefrontal brain function would be different in a homogenous sample. Future studies with more homogenous patient samples are therefore required; considering, however, that schizophrenia in itself is heterogeneous, and that many genetic studies investigate broad-spectrum schizophrenia with greater success than narrow-spectrum schizophrenia, it is unlikely that those studies would yield differing results.

In conclusion, the present data show a strong impact of COMT genotype on an electrophysiological correlate of cognitive response control and prefrontal functioning (NGA) in a group of patients with schizophrenia spectrum disorders. Carriers of the COMT allele related to a particularly high activity of the enzyme and thus shorter availability of dopamine in the synaptic cleft (Val) showed a significantly diminished NGA, indications of reduced fronto-central Nogo amplitudes, and an impaired Stroop performance, suggesting impaired functional activation of prefrontal structures probably including the ACC. These findings thus verify the impact of COMT Val158Met on prefrontal functioning and confirm the relevance of prefrontal dopaminergic tone for cognition. As the NGA was virtually absent in Val allele carriers, this might correspond to a genetically driven endophenotype in schizophrenic illnesses, possibly contributing to the cognitive deficits in schizophrenia. Strong genetic influences on prefrontal functioning might also account for the partly inconsistent results regarding a specifically frontal pathology ('hypofrontality') in schizophrenias, that is, for the highly variable symptomatology of disorders from the schizophrenic spectrum. Even though the present results need to be replicated in a larger sample and should also be confirmed by neuroimaging methods, neurophysiological approaches such as the present one are valuable tools in attempts to further elucidate the impact of genetic variations on cognitive functioning and functional brain activation in healthy subjects and neuropsychiatric disorders.

\section{ACKNOWLEDGEMENTS}

We gratefully acknowledge Theresa Töpner, Inge Gröbner, and Melanie Harder for excellent technical assistance. This study was supported by the Deutsche Forschungsgemeinschaft (Grant RE1632/1-1 to AR, KFO 125/1-1 to AR, AJF, and KPL, SFB 581 to KPL, and FA $361 / 8-1+2$ to 
AJF) and Bundesministerium für Bildung, Wissenschaft, Forschung und Technologie (IZKF, 01KS9603 to KPL).

\section{REFERENCES}

Abi-Dargham A, Moore H (2003). Prefrontal DA transmission at D1 receptors and the pathology of schizophrenia. Neuroscientist 9: 404-416.

Andreasen NC, Rezai K, Alliger R, Swayze VW, Flaum M, Kirchner $P$ et al (1992). Hypofrontality in neuroleptic-naïve patients and in patients with chronic schizophrenia. Assessment with xenon 133 single-photon emission computed tomography and the Tower of London. Arch Gen Psychiatry 49: 943-958.

Badner JA, Gershon ES (2002). Meta-analysis of whole-genome linkage scans of bipolar disorder and schizophrenia. Mol Psychiatry 7: 405-411.

Bilder RM, Volavka J, Czobor P, Malhotra AK, Kennedy JL, Ni X et al (2002). Neurocognitive correlates of the COMT Val(158)Met polymorphism in chronic schizophrenia. Biol Psychiatry 52: 701-707.

Bokura H, Yamaguchi S, Kobayashi S (2001). Electrophysiological correlates for response inhibition in a Go/NoGo task. Clin Neurophysiol 112: 2224-2232.

Carter CS, MacDonald AW, Ross LL, Stenger VA (2001). Anterior cingulate cortex activity and impaired self-monitoring of performance in patients with schizophrenia: an event-related fMRI study. Am J Psychiatry 158: 1423-1428.

Carter CS, Mintun M, Nichols T, Cohen JD (1997). Anterior cingulate gyrus dysfunction and selective attention deficits in schizophrenia: $\left[{ }^{15} \mathrm{O} \mathrm{H}_{2} \mathrm{O}\right.$ PET study during single-trial Stroop task performance. Am J Psychiatry 154: 1670-1675.

Chen X, Wang X, O’Neill AF, Walsh D, Kendler KS (2004). Variants in the catechol-o-methyltranserase (COMT) gene are associated with schizophrenia in Irish high-density families. Mol Psychiatry 9: 962-967.

Craddock N, Owen MJ, O'Donovan MC (2006). The catechol-Omethyl transferase (COMT) gene as a candidate for psychiatric phenotypes: evidence and lessons. Mol Psychiatry 11: 446-458.

de Zubicaray GI, Zelaya FO, Andrew C, Williams SC, Bullmore ET (2000). Cerebral regions associated with verbal response initiation, suppression and strategy use. Neuropsychologia 38: 1292-1304.

Egan MF, Goldberg TE, Kolachana BS, Callicott JH, Mazzanti CM, Straub RE et al (2001). Effect of COMT Val ${ }^{108 / 158}$ Met genotype on frontal lobe function and risk for schizophrenia. Proc Natl Acad Sci USA 98: 6917-6922.

Fallgatter AJ (2001). Electrophysiology of the prefrontal cortex in healthy controls and schizophrenic patients: a review. J Neural Transm 108: 679-694.

Fallgatter AJ, Bartsch AJ, Herrmann MJ (2002). Electrophysiological measurements of anterior cingulate function. J Neural Transm 109: 977-988.

Fallgatter AJ, Bartsch AJ, Zielasek J, Herrmann MJ (2003). Brain electrical dysfunction of the anterior cingulate in schizophrenic patients. Psychiatry Res 124: 37-48.

Fallgatter AJ, Brandeis D, Strik WK (1997). A robust assessment of the NoGo-anteriorisation of P300 microstates in a cued Continuous Performance Test. Brain Topogr 9: 295-302.

Fallgatter AJ, Herrmann MJ, Roemmler J, Ehlis A-C, Wagener A, Heidrich A et al (2004). Allelic variation of serotonin transporter function modulates the brain electrical response for error processing. Neuropsychopharmacology 29: 1506-1511.

Fallgatter AJ, Mueller TJ (2001). Electrophysiological signs of reduced prefrontal response control in schizophrenic patients. Psychiatry Res Neuroimaging 107: 19-28.

Ford JM, Gray M, Whitfield SL, Turken AU, Glover G, Faustman WO et al (2004). Acquiring and inhibiting prepotent responses in schizophrenia: event-related brain potentials and functional magnetic resonance imaging. Arch Gen Psychiatry 61: 119-129.

Gaillard WD, Hertz-Pannier L, Mott SH, Barnett AS, LeBihan D, Theodore WH (2000). Functional anatomy of cognitive development: fMRI of verbal fluency in children and adults. Neurology 54: 180-185.

Gallinat J, Bajbouj M, Sander T, Schlattmann P, Xu K, Ferro EF et al (2003). Association of the G1947A COMT (Val(108/ 158)Met) gene polymorphism with prefrontal P300 during information processing. Biol Psychiatry 54: 40-48.

Goldberg TE, Egan MF, Gscheidle T, Coppola R, Weickert T, Kolachana BS et al (2003). Executive subprocesses in working memory: relationship to catechol-O-methyltransferase Val158Met genotype and schizophrenia. Arch Gen Psychiatry 60: 889-896.

Gruber SA, Rogowska J, Holcomb P, Soraci S, Yurgelun-Todd D (2002). Stroop performance in normal control subjects: an fMRI study. Neuroimage 16: 349-360.

Hamilton M (1960). A rating scale for depression. J Neurol Neurosurg Psychiatry 23: 56-62.

Inada T, Nakamura A, Iijima Y (2003). Relationship between catechol-O-methyltransferase polymorphism and treatment-resistant schizophrenia. Am J Med Genet 120B: 35-39.

Ingvar DH, Franzen G (1974). Abnormalities of cerebral blood flow distribution in patients with chronic schizophrenia. Acta Psychiatr Scand 50: 425-462.

Jasper H (1958). Report of committee on methods of clinical exam in EEG. Electroencephalogr Clin Neurophysiol 10: 370-375.

Kay SR (1991). Positive and Negative Symptoms in Schizophrenia. Brunner/Mazel: New York.

Kiehl KA, Smith AM, Hare RD, Liddle PF (2000). An event-related potential investigation of response inhibition in schizophrenia and psychopathy. Biol Psychiatry 48: 210-221.

Kneavel M, Gogos J, Karayiorgou K, Luine V (2000). Interaction of COMT gene deletion and environment on cognition. Soc Neurosci Abstr 26: 571.20.

Kopp B, Rist F (1999). An event-related brain potential substrate of disturbed response monitoring in paranoid schizophrenic patients. J Abnorm Psychol 108: 337-346.

Laurens KR, Ngan ET, Bates AT, Kiehl KA, Liddle PF (2003). Rostral anterior cingulate cortex dysfunction during error processing in schizophrenia. Brain 126: 610-622.

Lehmann D (1987). Principles of spatial analysis. In: Gevins A, Remond A (eds). Methods of Analysis of Brain Electrical and Magnetic Signals. Handbook of Electroencephalography and Clinical Neurophysiology. Elsevier: Amsterdam. pp 309-354.

Lehmann D, Skrandies W (1980). Reference-free identification of components of checkerboard-evoked multichannel potential fields. Electroencephalogr Clin Neurophysiol 48: 609-621.

Mathalon DH, Fedor M, Faustman WO, Gray M, Askari N, Ford JM (2002). Response-monitoring dysfunction in schizophrenia: an event-related brain potential study. J Abnorm Psychol 111: $22-41$.

Matthews SC, Paulus MP, Simmons AN, Nelesen RA, Dimsdale JE (2004). Functional subdivisions within anterior cingulate cortex and their relationship to autonomic nervous system function. Neuroimage 22: 1151-1156.

Moll J, de Oliveira-Souza R, Moll FT, Bramati IE, Andreiuolo PA (2002). The cerebral correlates of set-shifting: an fMRI study of the trail making test. Arq Neuropsiquiatr 60: 900-905.

Nolan KA, Bilder RM, Lachman HM, Volavka J (2004). Catechol $O$-methyltransferase Val158Met polymorphism in schizophrenia: differential effects of Val and Met alleles on cognitive stability and flexibility. Am J Psychiatry 161: 359-361.

Overall JE, Gorham DR (1962). The brief psychiatric rating scale. Psychol Rev 10: 799-812.

Pascual-Marqui RD, Michel CM, Lehmann D (1994). Low resolution electromagnetic tomography, a new method for 
localizing electrical activity in the brain. Int J Psychophysiol 18: 49-65.

Reif A, Fallgatter AJ, Ehlis A-C, Lesch K-P (2004). Altered cingulate functioning in a case of chromosome 22q11 deletion syndrome. Psychiat Res Neuroimaging 132: 273-278.

Rubia K, Russell T, Bullmore ET, Soni W, Brammer MJ, Simmons A et al (2001). An fMRI study of reduced left prefrontal activation in schizophrenia during normal inhibitory function. Schizophr Res 52: 47-55.

Sanders AR, Rusu I, Duan J, Vander Molen JE, Hou C, Schwab SG et al (2005). Haplotypic association spanning the 22q11.21 genes COMT and ARVCF with schizophrenia. Mol Psychiatry 10: 353-365.

Strandburg RJ, Marsh JT, Brown WS, Asarnow RF, Guthrie D, Harper R et al (1999). Continuous-processing related ERPs in adult schizophrenia: continuity with childhood onset schizophrenia. Biol Psychiatry 45: 1356-1369.

Strik WK, Fallgatter AJ, Brandeis D, Pascual-Marqui R (1998). Three dimensional tomography of event-related potentials during response inhibition: evidence for phasic frontal lobe activation. Electroencephalogr Clin Neurophysiol 108: 406-413.

Sullivan PF, Kendler KS, Neale MC (2003). Schizophrenia as a complex trait: evidence from a meta-analysis of twin studies. Arch Gen Psychiatry 60: 1187-1192.

Tamminga CA, Thaker GK, Buchanan R, Kirkpatrick B, Alphs LD, Chase TN et al (1992). Limbic system abnormalities identified in schizophrenia with fluorodeoxyglucose and neocortical alterations with the deficit syndrome. Arch Gen Psychiatry 49: 522-530.

Tsai S-J, Yu YW-Y, Chen T-J, Chen J-Y, Liou Y-J, Chen M-C et al (2003). Association study of a functional catechol-Omethyltransferase-gene polymorphism and cognitive function in healthy females. Neurosci Lett 338: 123-126.

Tunbridge EM, Harrison PJ, Weinberger DR (2006). Catecholo-methyltransferase, cognition, and psychosis: $\mathrm{Val}^{158} \mathrm{Met}$ and beyond. Biol Psychiatry, print copy in press (originally published online Feb. 14, 2006, at www.sciencedirect.com ).

Volz H, Gaser C, Hager F, Rzanny R, Ponisch J, Mentzel H et al (1999). Decreased frontal activation in schizophrenics during stimulation with the continuous performance test - a functional magnetic resonance imaging study. Eur Psychiatry 14: 17-24.

Weinberger DR, Egan MF, Bertolino A, Callicott JH, Mattay VS, Lipska BK et al (2001). Prefrontal neurons and the genetics of schizophrenia. Biol Psychiatry 50: 825-844.

Weisbrod M, Kiefer M, Marzinzik F, Spitzer M (2000). Executive control is disturbed in schizophrenia: evidence from event-related potentials in a Go/NoGo task. Biol Psychiatry 47: 51-60.

Wonodi I, Stine OC, Mitchell BD, Buchanan RW, Thaker GK (2003). Association between $\mathrm{Val}^{108 / 158}$ Met polymorphism of the COMT gene and schizophrenia. Am J Med Genet 120B: $47-50$. 